\title{
Special Track on Worst Case Traversal Time (WCTT)
}

\author{
Anne Bouillard ${ }^{1}$, Marc Boyer ${ }^{2}$, Samarjit Chakraborty ${ }^{3}$, Steven Martin ${ }^{4}$, \\ Jean-Luc Scharbarg ${ }^{5}$, Giovanni Stea $^{6}$, and Eric Thierry ${ }^{7}$ \\ 1 ENS Cachan, France \\ 2 ONERA, France \\ 3 Technische Universität München, Germany \\ 4 LRI, France \\ 5 IRIT, France \\ ${ }^{6}$ University of Pisa, Italy \\ 7 ENS Lyon, France
}

Real-time systems are increasingly becoming communication intensive, where different functions are implemented using distributed real-time tasks mapped onto different physical systems (sensors, processors, and actuators). To ensure global correctness, one has to ensure the correctness of each task, schedulability of the tasks on each system, and finally also bound the communication time, i.e., the worst case (network) traversal time (WCTT).

Moreover, as systems grow bigger, connecting dozens to hundreds of systems, managing thousands of traffic flows, with each one interacting with the others, is becoming a challenging problem. This special track is devoted to formal methods on worst case traversal time in networks, with a special interest on scalable methods and on handling multi-hop networks.

Our conviction is that WCTT is an emerging area, made up of people from different communities, using different methods, and publishing in different conferences and/or journals.

People from several active research groups in this domain were contacted, and almost all of them have submitted a paper, despite the very short deadline. This reactivity confirms the desire of a community to find places to meet and discuss.

The aim of this track is to be the first meeting dedicated to WCTT, allowing us to capture a snapshot of this area, and also for fostering discussions on how this area should evolve. 